\title{
Validación de la Escala Subjetiva de Carga mental de trabajo (ESCAM) en profesionales de la salud de Chile*
}

Validation of the Subjective Mental Workload Scale (SCAM) in health professionals from Chile

Recibido: 28 de julio de 2014 | Aceptado: 01 de octubre de 2015

\author{
Paula Ceballos-Vásquez ** \\ Universidad Católica del Maule, Talca, Chile \\ GLADYS ROLO-GONZÁLEZ*** \\ ESTEFANÍA HERNÁNDEZ-FERNAUD \\ DOLORES DÍAZ-CABRERA \\ Universidad de La Laguna, Tenerife, España \\ Tatiana ParaviC-KLIJN ***** \\ Universidad de Concepción, Concepción, Chile \\ Mónica Burgos-MORENO ****** \\ OMAR BARRIGA \\ Universidad de Concepción, Concepción, Chile
}

doi : 10.11144/Javeriana.upsy15-1.vsmw

Para citar este artículo: Ceballos-Vásquez, P., RoloGonzález, G., Díaz-Cabrera, D., Paravic-Klijn, T., Burgos-Moreno, M., \& Barriga, O. (2016). Validación de la Escala Subjetiva de Carga mental de trabajo (ESCAM) en profesionales de la salud de Chile. Universitas Psychologica 15(1), 261-270.

http://dx.doi.org/10.11144/Javeriana.upsy15-1.vsmw

* Artículo de Investigación. Esta investigación contó con financiamiento del Plan de Mejoramiento Institucional en Oncología UCM1301, dependiente de la Universidad Católica del Maule, Talca, Chile.

** Doctora en Enfermería. Académica Departamento de Enfermería Facultad de Ciencias de la Salud.. Correo electrónico: pceballos@ucm.cl

**** Profesora titular de Universidad. Correo electrónico: grolog@ull.es; efernaud@ull.es Catedrática de Psicología Social. Correo electrónico: mddiaz@ull.es

***** Profesora Titular.Departamento de Enfermería. Facultad de Enfermería. Dra. en Enfermeria. Correo electrónico: tparavic@udec.cl

******** Profesora asociada Departamento de Enfermería. Facultad de Enfermería. Doctora en Enfermería. Correo electrónico: moni.burgos@gmail.com. Profesor asociado. $\mathrm{PhD}$ en Sociología. Correo electrónico: obarriga@udec.cl

\section{RES UMEN}

El objetivo de este estudio es confirmar las características psicométricas de la Escala Subjetiva de Carga Mental de Trabajo (ESCAM) en trabajadores sanitarios de hospitales chilenos. Participaron en este estudio 379 trabajadores de tres hospitales. Los instrumentos utilizados fueron: (a) ESCAM y (b) el cuestionario SUSESO-ISTAS 21. Los resultados indican que ESCAM se estructura en cinco dimensiones: Demandas cognitivas y complejidad de la tarea, Características de la tarea, Organización temporal, Ritmo de trabajo y Consecuencias para la salud. Asimismo, se observan correlaciones significativas entre la puntuación total de ESCAM y sus dimensiones con los factores de SUSESO-ISTAS 21. Además, se comprobó la validez de la escala mediante el análisis de perfiles de carga mental con diferentes grupos profesionales.

Palabras clave

carga mental de trabajo; escala subjetiva; salud ocupacional; validez; fiabilidad

\footnotetext{
A B S T R A C T

The aim of this study is to confirm the psychometric characteristics of the Subjective Mental Workload Scale (SCAM) in health workers of Chilean Hospitals. The instruments used were: (1) SCAM; (2) questionnaire SUSESO-ISTAS 21. The sample was composed of 379 workers from three hospitals. The findings showed that SCAM is structured in five dimensions: Cognitive demands and task complexity, Task characteristics, Temporal organization, Work rate, and Health consequences. Likewise, significant correlations between overall score of SCAM and its dimensions with the SUSESO-ISTAS 21 factors were found. Also, scale validity was verified through mental workload profiles with different professional groups. Keywords mental workload; subjective scale; occupational health; validity; reliability
} 
Paula Ceballos-Vásquez, Gladys Rolo-González, Estefanía Hernández-Fernaud, Dolores Díaz-Cabrera, Tatiana Paravic-Klijn, Mónica Burgos-Moreno, Omar Barriga

\section{Introducción}

Las exigencias mentales del trabajo, tanto por exceso como por defecto, tienen importantes consecuencias en el bienestar y la salud de las personas, así como sobre el logro de los objetivos de la organización, de ahí la relevancia de que sean evaluadas en los contextos de trabajo. (Díaz, Hernández, \& Rolo, 2012).

Un concepto estrechamente asociado a las exigencias mentales de los puestos de trabajo es el de carga mental, que se define como el esfuerzo cognitivo que debe realizar una persona en un tiempo concreto para hacer frente a determinada cantidad y tipos de tareas (Díaz, Fernández \& Rolo, 2012; Ferrer \& Dalmau, 2014; Gaillard, 2001). Este concepto parte de una serie de premisas: (a) los recursos cognitivos-atencionales de las personas son limitados; (b) la relevancia de los criterios de ejecución objetivos y subjetivos, tanto los establecidos por fuentes externas como por el propio trabajador, $y$ (c) la influencia en la carga mental de las demandas y características de la tarea (p.ej., concentración, atención, memoria), de las variables individuales (p.ej., nivel de experiencia) y de otras variables externas de la situación (p.ej., las ayudas tecnológicas).

A pesar del interés mostrado desde la investigación básica y aplicada por la carga mental, la diversidad de variables intervinientes en la misma y de modelos teóricos explicativos desde los que se intenta abordar su estudio, ha hecho difícil contar con una definición ampliamente consensuada. En un intento por unificar las definiciones existentes, Hacker (2001) plantea dos grandes enfoques de análisis de la carga mental de trabajo. El primer enfoque, denominado exigenciasrecursos, se centra en los procesos cognitivos-atencionales sin considerar la interacción entre las demandas de la tarea y los recursos del trabajador. En el segundo enfoque, denominado exigencias de la tarea, se considera la interacción entre las características de la tarea, del trabajador y del contexto.

La Norma ISO-10075-1 parte del segundo enfoque propuesto por Hacker (2001) y asume que la carga mental está determinada por las demandas externas que la persona debe afrontar con sus características y recursos personales, incluyendo capacidades cognitivas, emocionales y sociales. Esta norma considera que la carga mental es el resultado de la presión mental que producen en el trabajador los factores externos a la tarea (p.ej., complejidad, presión temporal). Esta presión genera un nivel de tensión mental que dependerá de las características de la persona, y que provoca consecuencias sobre su salud (Díaz, Hernández, Rolo, Galván, Fraile \& Loayssa, 2010).

Los factores causales de carga mental según esta Norma ISO se refieren al entorno y a las relacionadas con el individuo. Además, se consideran efectos y consecuencias asociadas a la carga mental. En cuanto a los factores causales del entorno, destacan las características de las tareas (p.ej., complejidad y cantidad de tareas, demandas de toma de decisiones, ritmo de trabajo); las condiciones físicoambientales (p.ej., ruido, iluminación, temperatura o distribución del espacio de trabajo); los factores sociales y de la organización (p.ej., mecanismos de coordinación, grado de formalización y estandarización, nivel de especialización de los puestos), y los factores extralaborales (p.ej., situación económica general y valores culturales). Respecto a los factores causales del individuo, la norma considera aspectos como las aptitudes y habilidades del trabajador, sus conocimientos y experiencia, necesidades personales, motivación, salud y nivel de activación.

En relación con los efectos y consecuencias, el modelo incluye efectos inmediatos de la carga mental, facilitadores (p.ej., calentamiento, activación) o perjudiciales (p.ej., fatiga mental, monotonía). Los efectos perjudiciales se deben al desequilibrio entre las demandas de las tareas y las características del trabajador y pueden producir dos niveles de carga mental: sobrecarga o subcarga. La sobrecarga hace referencia a las circunstancias en las que el trabajador se ve sometido a más exigencias mentales de las que puede responder en función de sus capacidades, conocimientos y experiencia. Mientras que la subcarga mental de trabajo emerge cuando las tareas a realizar por el trabajador son escasas y tienen un bajo nivel de exigencia mental. Las consecuencias a largo plazo de las situaciones extremas de carga mental incluyen riesgos psicosociales como estrés, síndrome de quemarse por el trabajo o mobbing (Meseguer de Pedro, Soler, García-Izquierdo, Sáez, \& Sánchez, 2007; Patlán, 2013). 
El constructo de carga mental de trabajo es de naturaleza multidimensional, lo que conlleva una pluralidad metodológica para su evaluación. Así, se han desarrollado diferentes procedimientos de evaluación clasificados en medidas fisiológicas (p.ej., tasa cardíaca), medidas basadas en el rendimiento (p.ej., medidas de tarea múltiple), y medidas subjetivas (p.ej., NASA-TLX). Las medidas subjetivas permiten obtener una valoración de las personas sobre el nivel de carga mental experimentado durante la realización de una tarea, asumiendo que esta valoración se relaciona con las exigencias objetivas de la misma. Este tipo de medidas tiene un bajo nivel de intrusión, son fáciles de utilizar, tienen aceptación por parte de los trabajadores y pocos requisitos de implementación (Díaz, Hernández et al., 2012).

Este estudio utiliza la Escala Subjetiva de Carga mental de trabajo (ESCAM) desarrollada por Rolo, Díaz \& Hernández (2009), que es una escala multidimensional para evaluar la carga mental percibida del puesto de trabajo. Esta escala se basa en una valoración global de las tareas desempeñadas en los puestos de trabajo. La elaboración de esta escala incluye factores relativos a las características de las tareas y condiciones temporales de trabajo, así como efectos perjudiciales de la carga, concretamente síntomas de fatiga mental.

El objetivo de este trabajo es confirmar las características psicométricas de la ESCAM en trabajadores sanitarios de Unidades de Pacientes Críticos (UPC) de hospitales chilenos. El estudio de la carga mental en estas unidades es importante porque concurren factores causales de carga mental relevantes. Así, este contexto de trabajo exige atención constante, memorización de información, toma de decisiones inmediatas y críticas, así como trabajar con personas en estado grave, pudiendo generar desgaste emocional (Castillo, Torres, Ahumada, Cárdenas, \& Licona, 2014; Novoa et al., 2005).

\section{Método}

\section{Participantes}

En esta investigación participaron 379 trabajadores de UPC de tres hospitales chilenos (38.8\%, 26.9\% y $34.3 \%$ de la muestra), que corresponde al $86.9 \%$ del total de trabajadores de UPC. No se recogieron datos del $13.1 \%$ restante por encontrarse con licencia o ausentes de las respectivas organizaciones durante el periodo de recolección de los datos o por no querer participar. El 59.1\% de los participantes trabajan en unidades de cuidados intensivos y el 40.9\% restante en unidades de cuidados intermedios. Los participantes ocupan puestos de médico-a (14.5\%), enfermero-a (29.3\%), fisioterapeuta (4.7\%), técnicos de enfermería (TENS) (38.3\%), auxiliar de servicio $(11.3 \%)$, administrativo-a $(1.6 \%)$ y nutricionista $(0.3 \%)$.

El 48\% de los participantes trabajan con contrato determinado (plazo fijo), el 30.1\% tienen un contrato indeterminado (plazo temporal) y el $21.9 \%$ tienen otro tipo de contrato. El 83.4\% trabajan en turnos rotatorios. La antigüedad media en la institución hospitalaria es 10.09 años (d.t. $=9.79$, $\min =$ 0, máx $=38$ ) y la mediade antigüedad en el puesto actual es de 7.38 años $($ d.t. $=7.75 \min =0$, $\operatorname{máx}=35)$.

La edad media de los trabajadores es de 37.36 años (d.t.= 10.53; Rango: 21-64). El 72.3\% de la muestra son mujeres y el $27.7 \%$ varones. El $76.1 \%$ de los participantes manifiestan tener pareja. En cuanto al nivel educativo, el $50.1 \%$ posee estudios universitarios, el $42.2 \%$ tiene estudios de nivel técnico y el $7.7 \%$ tiene estudios medios.

\section{Instrumentos}

En esta investigación se utilizaron dos instrumentos que se describen a continuación:

1) Escala Subjetiva de Carga Mental de Trabajo (ESCAM): esta escala fue desarrollada por Rolo et al. (2009) para la valoración subjetiva de Carga Mental de Trabajo. Está compuesta por 20 ítems que constituyen cinco factores: (a) Demandas cognitivas y complejidad de la tarea, que hace alusión al esfuerzo mental que supone el desempeño del puesto de trabajo; (b) Características de la tarea, que tiene que ver con las interrupciones o distracciones que sufre el trabajador en su puesto; (c) Organización temporal del trabajo, que recoge las valoraciones sobre la adecuación del tiempo del que disponen los trabajadores para realizar las tareas; (d) Ritmo 
Paula Ceballos-Vásquez, Gladys Rolo-González, Estefanía Hernández-Fernaud, Dolores Díaz-Cabrera, Tatiana Paravic-Klijn, Mónica Burgos-Moreno, Omar Barriga

de trabajo, que se refiere a la organización y planificación del tiempo de trabajo por parte del trabajador, y la probabilidad de cometer errores, y (e) Consecuencias para la salud, que hace referencia al agotamiento que produce el desempeño del puesto en el ocupante. Las puntuaciones oscilan entre 1 y 5 , siendo 1 baja carga mental y 5 alta. La escala permite obtener el promedio de carga mental subjetiva y puntuaciones específicas para cada una de las dimensiones.

2) Cuestionario SUSESO-ISTAS 21 (versión breve): este instrumento permite valorar las repercusiones de varios factores psicosociales en la salud del trabajador. Está formado por 20 ítems que se agrupan en cinco dimensiones: (a) Exigencias psicológicas de tipo cognitivo, cuantitativo, emocional y sensorial; (b) Trabajo activo y desarrollo de habilidades, que se refiere a la autonomía, el sentido del trabajo y las oportunidades de desarrollo del trabajador; (c) Apoyo social en la empresa y calidad de liderazgo, que incluye claridad de rol y conflicto de rol, características del liderazgo ejercido y apoyo socioinstrumental por parte de los superiores o compañeros; (d) Compensaciones, que engloba aspectos relacionados con el reconocimiento percibido y la inseguridad respecto a las condiciones laborales, $y$ (e) Doble presencia, que alude a la cantidad de trabajo doméstico o familiar que depende del trabajador. Este cuestionario permite obtener el nivel de riesgo percibido por los trabajadores. Este instrumento se encuentra adaptado, validado y estandarizado para Chile por la Superintendencia de Salud (Alvarado et al., 2012).

\section{Procedimiento}

La aplicación de los instrumentos se realizó mediante una entrevista individual entre los meses de mayo y octubre de 2013. La duración de cada entrevista fue de 15 a 20 minutos. Los trabajadores participaron voluntariamente, previa firma de consentimiento libre e informado. Para la realización del estudio se obtuvo la aprobación del Comité de Bioética de la Facultad de Medicina de la Universidad de Concepción (Chile) y del comité Ético-Científico de las instituciones de salud participantes.

\section{Resultados}

En primer lugar se analizó la estructura factorial en la que se organizan los ítems de la Escala Subjetiva de Evaluación de Carga Mental (ESCAM), así como su consistencia interna. En segundo lugar, se estudió la validez del instrumento siguiendo dos procedimientos: a) analizando la correlación entre las puntuaciones de Carga mental con los Factores psicosociales evaluados con SUSESO-ISTAS 21, y b) mediante un procedimiento de known-group validity (Zeller \& Carmines, 1980).

\section{Análisis factorial exploratorio: estructura empírica de la Escala Subjetiva de Evaluación de la Carga Mental (ESCAM)}

Como paso previo al análisis factorial, se realizó una depuración estadística de los ítems. Se comprobó la ausencia de outliers univariados mediante las puntuaciones típicas extremas y la capacidad de discriminación de los ítems (correlación entre el ítem y el total de la escala excluido el ítem). Se contrastó la existencia de outliers multivariados con la distancia de Mahalanobis, eliminándose tres casos por presentar outliers (Tabachnick \& Fidell, 1989).

Se realizó un análisis factorial de componentes principales y rotación ortogonal (Varimax). El coeficiente de adecuación muestral KMO fue 0.780 y el valor de la prueba de esfericidad de Bartlett fue $\chi^{2}(190)=1846.607, p \leq 0.001$. La matriz de correlaciones anti-imagen mostró que los valores MSA por cada ítem eran superiores a 0.60 y el $88.25 \%$ de las puntuaciones era inferior a 0.1 . Se consideraron los factores con valor propio superior a uno y los ítems con saturaciones a partir de 0.30 (Tabla 1).

La solución factorial obtenida agrupó los ítems en cinco factores, al igual que en la estructura presentada por Rolo et al., (2009), que explican el $54.49 \%$ de la varianza. El alfa de Cronbach para el total de la escala es de 0.73 , siendo tanto este valor como los obtenidos en cada factor muy similares a los alcanzados en el trabajo original.

El primer factor explica el 19.59\% de la varianza y representa la dimensión Demandas cognitivas y complejidad de la tarea, relativa al esfuerzo cogniti- 
TABLA 1.

Factores y pesos factoriales de los ítems, correlación ítem-factor, consistencia interna de los factores y del factor eliminando el elemento

\begin{tabular}{|c|c|c|c|}
\hline$\overline{\text { Factores e ítems }}$ & Peso & $\begin{array}{l}\text { R ítem- } \\
\text { factor }\end{array}$ & $\begin{array}{c}\text { Alfa sin } \\
\text { ítem }\end{array}$ \\
\hline $\begin{array}{l}\text { FACTOR 1. DEMANDAS COGNITIVAS Y COMPLEJIDAD DE TAREA } \alpha=0.77 \\
\text { 1. El grado de complejidad de la información que debo utilizar en mi trabajo es } \\
\text { 2. La cantidad de memorización de información y material que requiere mi trabajo es } \\
\text { 4. El nivel de esfuerzo o concentración mental que requiere mi trabajo es } \\
\text { 8.El nivel de esfuerzo mental necesario para evitar los errores en mi trabajo es } \\
\text { 4. Habitualmente en mi puesto de trabajo el número de decisiones que debo tomar es }\end{array}$ & $\begin{array}{l}0.82 \\
0.80 \\
0.75 \\
0.59 \\
0.55\end{array}$ & $\begin{array}{l}0.59 \\
0.63 \\
0.65 \\
0.44 \\
0.47\end{array}$ & $\begin{array}{l}0.71 \\
0.70 \\
0.71 \\
0.77 \\
0.76\end{array}$ \\
\hline $\begin{array}{l}\text { FACTOR 2. ORGANIZACIÓN TEMPORAL } \alpha=0.78 \\
\text { 19.El tiempo de que dispongo para realizar mi trabajo es } \\
\text { 20. El tiempo asignado a cada una de las tareas que realizo es } \\
\text { 21. El tiempo del que dispongo para tomar las decisiones exigidas por mi trabajo es }\end{array}$ & $\begin{array}{l}0.87 \\
0.85 \\
0.67\end{array}$ & $\begin{array}{l}0.48 \\
0.72 \\
0.67\end{array}$ & $\begin{array}{l}0.84 \\
0.59 \\
0.65\end{array}$ \\
\hline $\begin{array}{l}\text { FACTOR 3. CONSECUENCIAS PARA LA SALUD } \alpha=0.70 \\
\text { 16. Me siento agotado cuando me levanto por la mañana y tengo que enfrentarme a } \\
\text { otro día de trabajo } \\
\text { 17.Tengo dificultades para relajarme después del trabajo } \\
\text { 15. Al final de la jornada de trabajo me siento agotado } \\
\text { 9. El cansancio que me produce mi trabajo es }\end{array}$ & $\begin{array}{l}0.76 \\
0.73 \\
0.73 \\
0.70\end{array}$ & $\begin{array}{l}0.48 \\
0.50 \\
0.58 \\
0.51\end{array}$ & $\begin{array}{l}0.67 \\
0.65 \\
0.58 \\
0.66\end{array}$ \\
\hline \multirow{2}{*}{$\begin{array}{l}\text { FACTOR 4. CARACTERÍSTICAS DE LA TAREA } \alpha=0.57 \\
\text { 6. El número de interrupciones (llamadas telefónicas, atender público, otros compa- } \\
\text { neros solicitando información, etc.) durante la realización de mi trabajo es } \\
\text { 10. Las tareas que realizo en mi trabajo requieren una alta concentración debido a la } \\
\text { cantidad de distracción o ruido de fondo } \\
\text { 7. La cantidad de dificultades que se producen cuando se introducen nuevos procedi- } \\
\text { mientos de trabajo o programas informáticos es } \\
\text { 5. El nivel de ambigüedad de las decisiones a tomar en mi trabajo es } \\
\text { 13. En mi trabajo tengo que hacer más de una tarea a la vez }\end{array}$} & 0.65 & 0.41 & 0.47 \\
\hline & $\begin{array}{l}0.61 \\
0.59 \\
0.39\end{array}$ & $\begin{array}{l}0.40 \\
0.31 \\
0.24\end{array}$ & $\begin{array}{l}0.49 \\
0.53 \\
0.57\end{array}$ \\
\hline $\begin{array}{l}\text { FACTOR 5. RITMO DE TRABAJO } \alpha=0.39 \\
\text { 11. Es posible variar mi ritmo de trabajo sin perturbar el trabajo de mi sección } \\
\text { 14. En mi trabajo puedo cometer algún error sin que incida en forma crítica sobre los }\end{array}$ & 0 & 0.30 & 0.15 \\
\hline $\begin{array}{l}\text { resultados del trabajo } \\
12 \text {. Además de las pausas reglamentarias, el trabajo me permite hacer alguna pausa } \\
\text { cuando lo necesito }\end{array}$ & $\begin{array}{l}0.68 \\
0.38\end{array}$ & $\begin{array}{l}0.19 \\
0.20\end{array}$ & $\begin{array}{l}0.38 \\
0.36\end{array}$ \\
\hline
\end{tabular}

Fuente: elaboración propia

vo que requiere el desempeño del puesto de trabajo. Este factor tiene un alfa de Cronbach de 0.77 y está compuesto por cinco ítems, a diferencia del factor obtenido por Rolo et al., (2009) que incluía seis ítems. El ítem 5, "el nivel de ambigüedad de las decisiones a tomar en mi trabajo es", saturó en el factor Característica de la tarea. Esta diferencia no favorece ni perjudica la consistencia interna de ningún factor.

Los dos siguientes factores están compuestos por los mismos ítems que los expuestos en la estructura original. El factor dos, Organización temporal, está formado por tres ítems que valoran la disponibilidad de tiempo para desempeñar el trabajo. Este factor, que explica el $11.84 \%$ de la varianza, tiene una consistencia interna de 0.78. El factor tres,
Consecuencias para la salud, incluye cuatro ítems respecto a los síntomas de fatiga mental que experimenta el trabajador por la realización del trabajo, explica el $10.18 \%$ de la varianza y tiene un alfa de Cronbach de 0.70 .

El cuarto factor, Características de las tareas, explica el $7.38 \%$ de la varianza e incorpora un ítem a los cuatro presentados por Rolo et al., (2009) en este factor. La consistencia interna de este factor fue 0.57. Como ya se mencionó, se añade un ítem que originalmente estaba incluido en el factor Demandas cognitivas y complejidad de la tarea (ítem 5), de forma que el factor describe las interrupciones y distracciones que sufre el trabajador, así como la dificultad y la ambigüedad en el puesto. 
Por último, el factor Ritmo de trabajo se comporta igual que el original. Incluye tres ítems referidos a la posibilidad que tiene el trabajador de distribuir el tiempo para realizar sus tareas y a las consecuencias que tiene un error. Este factor explica el 5.50\% de la varianza y tiene una consistencia interna de 0.39 .

\section{Validez de la Escala Subjetiva de}

Carga mental: análisis de correlaciones

$y$ análisis de perfiles de carga

mental en función del puesto

En la Tabla 2 se presentan los cálculos de las puntuaciones para cada uno de los factores de Carga mental y de Carga mental global. Se observa que las puntuaciones de Carga mental son superiores al punto medio de la escala de respuesta en todas las dimensiones, excepto Organización temporal y en la puntuación global de Carga. Destacan como puntuaciones más altas las de los factores Demandas cognitivas y complejidad de la tarea, Características de la tarea y Consecuencias para la salud.

Para analizar la validez de la escala ESCAM se analizaron, en primer lugar, las correlaciones de dicho instrumento con SUSESO-ISTAS 21, que incluye una dimensión relativa a las exigencias cognitivas y emocionales que tiene el puesto de trabajo y que, conceptualmente, evalúa algunos aspectos de Carga mental. En la Tabla 2 se observa que existen correlaciones positivas y significativas entre la puntuación global de Carga mental y todas las dimensiones psicosociales de SUSESO-ISTAS 21, de forma que cuando los trabajadores realizan una valoración negativa de los factores psicosociales medidos, también perciben una Carga mental de trabajo mayor. Destaca que la dimensión psicosocial Exigencias psicológicas correlaciona de forma

TABLA 2.

Estadísticos descriptivos y correlaciones entre Carga mental y Factores de riesgos psicosociales

\begin{tabular}{|c|c|c|c|c|c|c|c|c|c|c|c|c|c|}
\hline & \multicolumn{8}{|c|}{ ESCAM } & \multicolumn{4}{|c|}{ SUSESO-ISTAS 21} \\
\hline & & M & $\mathrm{dt}$ & 1 & 2 & 3 & 4 & 5 & 6 & 7 & 8 & 9 & 10 \\
\hline & ESCAM & & & & & & & & & & & & \\
\hline 1 & Carga mental global & 3.39 & 0.43 & & & & & & & & & & \\
\hline 2 & $\begin{array}{l}\text { Demandas cognitivas } \\
\text { y complejidad de la } \\
\text { tarea }\end{array}$ & 3.79 & 0.57 & $0.524 * *$ & & & & & & & & & \\
\hline 3 & $\begin{array}{l}\text { Organización } \\
\text { temporal del trabajo }\end{array}$ & 2.73 & 0.76 & $0.658^{* *}$ & $0.174 * *$ & & & & & & & & \\
\hline 4 & $\begin{array}{l}\text { Consecuencias para } \\
\text { la salud }\end{array}$ & 3.67 & 0.85 & $0.560 * *$ & 0.094 & $0.148^{* *}$ & & & & & & & \\
\hline 5 & $\begin{array}{l}\text { Características de la } \\
\text { tarea }\end{array}$ & 3.70 & 0.66 & $0.619^{* *}$ & $0.426 * *$ & $0.281 * *$ & $0.216^{* *}$ & & & & & & \\
\hline 6 & Ritmo de trabajo & 3.03 & 0.85 & $0.545^{* *}$ & 0.074 & $0.285^{* *}$ & 0.047 & 0.032 & & & & & \\
\hline & SUSESO- ISTAS 21 & & & & & & & & & & & & \\
\hline 7 & $\begin{array}{l}\text { Exigencias } \\
\text { Psicológicas }\end{array}$ & 11.78 & 2.79 & $0.480^{* *}$ & $0.394 * *$ & $0.245^{* *}$ & $0.266^{* *}$ & $0.377^{* *}$ & $0.171 * *$ & & & & \\
\hline & $\begin{array}{l}\text { Trabajo activo } \\
\text { y desarrollo de } \\
\text { habilidades }\end{array}$ & 6.77 & 2.54 & $0.268 * *$ & -0.019 & $0.218^{* *}$ & $0.233 * *$ & $0.175^{* *}$ & $0.125^{*}$ & $0.188^{* *}$ & & & \\
\hline 9 & $\begin{array}{l}\text { Apoyo social en la } \\
\text { empresa y calidad de } \\
\text { liderazgo }\end{array}$ & 6.50 & 2.85 & $0.268 * *$ & -0.030 & $0.223 * *$ & $0.292 * *$ & $0.178 * *$ & 0.067 & $0.294 * *$ & $0.369^{* *}$ & & \\
\hline 10 & Compensaciones & 4.70 & 3.03 & $0.244 * *$ & 0.005 & $0.137 * *$ & $0.322 * *$ & $0.147^{* *}$ & 0.054 & 0.028 & $0.237^{* *}$ & $0.290 * *$ & \\
\hline & Doble presencia & 3.64 & 2.10 & $0.318^{* *}$ & 0.041 & $0.191 * *$ & $0.326 * *$ & $0.200 * *$ & $0.123 *$ & $0.198 * *$ & $0.162 * *$ & $0.236 * *$ & $0.238 * *$ \\
\hline
\end{tabular}

$* \mathrm{p}<0.05 ; * \mathrm{p}<0.01$

Fuente: elaboración propia 
positiva y significativa con todas las dimensiones de Carga mental de trabajo, siendo la correlación más alta con el factor Demandas cognitivas y complejidad de la tarea (0.39) y la más baja con el factor Ritmo de trabajo (0.17).

En segundo lugar se utilizó un procedimiento de Known-group validity (Zeller \& Carmines, 1980), comparando los perfiles de Carga mental de distintos grupos de trabajo. Para ello, se consideraron cuatro grupos de profesionales: médicos $(n=55)$, enfermeros $(n=111)$, técnicos de enfermería (TENS) $(n=145)$ y auxiliares de servicio $(n=43)$, ya que son los de mayor tamaño. Se realizó un análisis de perfiles mediante análisis de varianza de medidas repetidas con 354 trabajadores. La variable intersujeto fue grupos de trabajo, con cuatro niveles, y la variable intrasujeto el perfil de Carga mental de trabajo, con cinco niveles correspondientes a los factores de dicha variable.

La homogeneidad multivariada de las varianzas se comprobó con la prueba $\mathrm{M}$ de Box que resultó significativa $(F(45,89653)=1.80, p \leq 0.001)$, por lo que se utilizó la Lambda de Wilks con criterio Pillai (Tabachnick \& Fidell, 1989). Se obtuvo un efecto principal de la variable grupos de trabajo
$\left(F(3,350)=7.39, p \leq 0.001, \eta^{2}=0.06\right)$ y un efecto principal del perfil de Carga Mental $(F(4,347)=$ 124.72, $p \leq 0.001, \eta^{2}=0.59$ ). Además, se obtuvo una interacción significativa entre ambas variables $\left(F(12,1047)=7.52, p \leq 0.001, \eta^{2}=0.08\right)($ Figura 1$)$.

Los análisis a posteriori de la interacción entre la variable intersujeto de trabajo y la intrasujeto perfil de Carga mental se presentan en la Tabla 3. Destaca que la prueba a posteriori de Scheffe mostró que, en conjunto, el perfil de Carga mental de los auxiliares de servicio es significativamente menor que el de médicos, enfermeros y técnicos de enfermería $(p \leq 0,01)$.

Se obtuvo que en la dimensión Demandas cognitivas y complejidad de la tarea existen diferencias significativas entre todos los grupos de trabajo, excepto entre médicos y enfermeros. En relación al factor Organización temporal, no hay diferencias estadísticamente significativas entre los grupos de trabajo. Se observa también que los técnicos en enfermería (TENS) obtienen puntuaciones significativamente más altas que médicos, enfermeros y auxiliares de servicios en la dimensión Consecuencias para la salud. En la dimensión Características de la tarea destaca

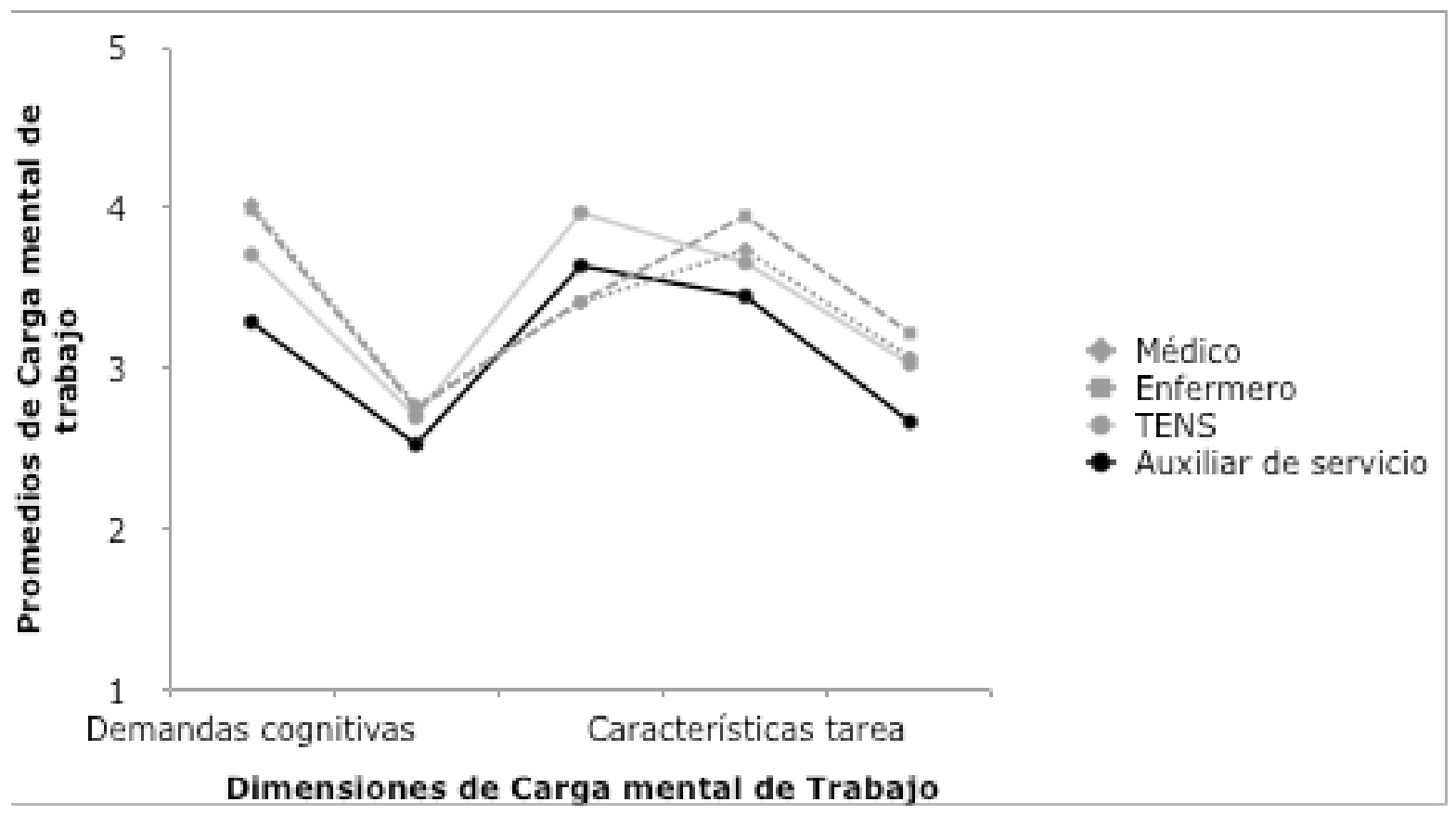

Figura 1. Perfil de Carga mental en función del grupo de trabajo Fuente: elaboración propia 
Paula Ceballos-Vásquez, Gladys Rolo-González, Estefanía Hernández-Fernaud, Dolores Díaz-Cabrera, Tatiana Paravic-Klijn, Mónica Burgos-Moreno, Omar Barriga

TABLA 3.

Contrastes a posteriori entre grupos de trabajo para cada factor de Carga mental

\begin{tabular}{lccccc}
\hline & $\begin{array}{c}\text { Demandas } \\
\text { cognitivas }\end{array}$ & $\begin{array}{c}\text { Organización } \\
\text { temporal }\end{array}$ & $\begin{array}{c}\text { Consecuencias } \\
\text { salud }\end{array}$ & $\begin{array}{c}\text { Características } \\
\text { tarea }\end{array}$ & Ritmo \\
\hline Médicos - Enfermeros & $n . s$. & $n . s$. & $n . s$. & $t=-2.04^{*}$ & $n . s$. \\
Médicos - TENS & $t=3.86^{* * *}$ & $n . s$. & $t=-4.25^{* * *}$ & $n . s$. & $n . s$. \\
Médicos - Auxiliares & $t=6.88^{* * *}$ & $n . s$. & $n . s$. & $t=2.16^{*}$ & $t=2.31^{*}$ \\
Enfermeros - TENS & $t=4.36^{* * *}$ & $n . s$. & $t=-5.30^{* * *}$ & $t=3.61^{* * *}$ & $n . s$. \\
Enfermeros - Auxiliares & $t=7.45^{* * * *}$ & $n . s$. & $n . s$. & $t=4.32^{* * * *}$ & $t=3.64^{* * * *}$ \\
TENS - Auxiliares & $t=4.55^{* * *}$ & $n . s$. & $t=2.25^{*}$ & $n . s$. & $t=2.47^{*}$ \\
\hline
\end{tabular}

Fuente: elaboración propia

la puntuación más alta obtenida por los enfermeros frente a los restantes grupos de trabajo. Y por último, en el factor Ritmo de trabajo los auxiliares de servicios puntúan más bajo que los enfermeros, médicos y TENS. En la Tabla 4 se presentan las medias y desviaciones típicas en cada dimensión de Carga mental para cada grupo de trabajo.

\section{Discusión}

El objetivo de este estudio es confirmar las características psicométricas de la Escala Subjetiva de Carga Mental de Trabajo (ESCAM) en profesionales de la salud en Unidades de Pacientes Críticos (UPC) en Chile. Este estudio tiene un alto interés porque responde a la necesidad de comprobar la estructura de la escala en otra cultura y en organizaciones con estructuras, procesos organizacionales y cultura organizacional diferentes a la española (Gómez-Ortiz, 2011). Estas diferencias deben ser tenidas en cuenta, pues pueden afectar la percepción de carga mental, así como la estructura empírica de la escala. Asimismo, las características de estos puestos de trabajo con altas demandas cognitivas, presión temporal, toma de decisiones complejas, solapamiento de tareas y desgaste emocional, entre otros aspectos (Castillo et al., 2014; Novoa et al., 2005), justifican la relevancia de contar con un instrumento validado en este grupo de trabajadores-as.

Los resultados obtenidos en esta investigación muestran que la escala de carga mental se estructura en cinco dimensiones que coinciden casi en su totalidad con la estructura original (Rolo et al., 2009), y con el estudio preliminar realizado por Ceballos, Paravic, Burgos y Barriga (2014). Así, se identifican los factores Demandas cognitivas y complejidad de la tarea, Característica de la tarea, Organización temporal, Ritmo de trabajo y Consecuencias para la salud. La consistencia interna del total de la escala es alta, así como la de tres

TABLA 4.

Estadísticos descriptivos de las dimensiones de Carga mental por grupos de trabajo

\begin{tabular}{lccccccccccc}
\hline & \multicolumn{2}{c}{$\begin{array}{c}\text { Demandas } \\
\text { cognitivas }\end{array}$} & \multicolumn{2}{c}{$\begin{array}{c}\text { Organización } \\
\text { temporal }\end{array}$} & \multicolumn{2}{c}{$\begin{array}{c}\text { Consecuencias } \\
\text { salud }\end{array}$} & \multicolumn{2}{c}{$\begin{array}{c}\text { Características } \\
\text { tarea }\end{array}$} & \multicolumn{2}{c}{ Ritmo } \\
\hline & $\mathrm{M}$ & $\mathrm{dt}$ & $\mathrm{M}$ & $\mathrm{dt}$ & $\mathrm{M}$ & $\mathrm{dt}$ & $\mathrm{M}$ & $\mathrm{dt}$ & $\mathrm{M}$ & $\mathrm{dt}$ \\
\hline Médicos & 4.02 & 0.41 & 2.77 & 0.83 & 3.41 & 0.76 & 3.74 & 0.59 & 3.07 & 0.85 \\
Enfermeros & 3.99 & 0.46 & 2.75 & 0.71 & 3.42 & 0.86 & 3.95 & 0.56 & 3.22 & 0.79 \\
TENS & 3.71 & 0.57 & 2.70 & 0.76 & 3.97 & 0.79 & 3.66 & 0.71 & 3.03 & 0.86 \\
Auxiliares de servicio & 3.29 & 0.55 & 2.53 & 0.79 & 3.64 & 0.89 & 3.45 & 0.65 & 2.67 & 0.94 \\
\hline
\end{tabular}

Fuente: elaboración propia 
de los factores obtenidos (Demandas cognitivas y complejidad de la tarea, Organización temporal y Consecuencias para la salud). Los factores Características de la tarea y Ritmo de trabajo muestran menor consistencia interna, lo que indica que estas dimensiones requieren una revisión de los ítems que componen la escala. Estos valores van en la misma línea de los encontrados en el trabajo original.

La validez de la escala se comprobó mediante dos procedimientos. En primer lugar, se realizó la correlación del total de la escala de carga mental y de sus cinco factores con los factores psicosociales contenidos en la escala SUSESO-ISTAS 21. Se observan correlaciones significativas entre la puntuación total de ESCAM y sus dimensiones con los factores de SUSESO-ISTAS 21, destacando especialmente las altas correlaciones con el factor psicosocial Exigencias psicológicas. Estas correlaciones indican que ESCAM está midiendo uno de los factores principales de carga mental de trabajo: los requerimientos cognitivos de las tareas. El factor de carga mental Consecuencias para la salud, muestra correlaciones más altas con Doble presencia y Compensaciones de SUSESO-ISTAS 21. Esto se explica considerando que la doble presencia es un factor negativo que incide de forma directa en la fatiga mental. Mientras que las compensaciones, al ser un motivador extrínseco, pueden modular la percepción de carga mental y, por lo tanto, influir en la salud del trabajador.

En segundo lugar, se comprobó la validez de la escala mediante el análisis de perfiles de carga mental con diferentes grupos de profesionales. El perfil global de carga mental de los auxiliares de servicio es significativamente distinto que el de los restantes puestos de trabajo analizados. En este sentido, perciben menores demandas cognitivas y ritmo de trabajo, y valoran más negativamente las características de la tarea y la organización temporal.

En relación con cada uno de los factores, destacan las puntuaciones altas de los técnicos en enfermería en síntomas de fatiga, las mayores demandas cognitivas y atencionales en médicos y enfermeros, y la cantidad de interrupciones y distracciones presentes en el desempeño del puesto de enfermero. Estos resultados son coherentes con las caracterís- ticas de estos trabajos. Los técnicos en enfermería desempeñan muchas tareas diferentes, algunas de ellas con carga física, lo que puede provocar mayor percepción de fatiga. Los puestos de médicos y enfermeros se caracterizan, entre otros aspectos, por toma de decisiones críticas y rápidas, altas demandas de concentración, atencionales y de memoria. El puesto de enfermería requiere, entre otros, comunicación continuada con otros profesionales y usuarios de los servicios, vigilancia y monitorización del paciente, y atención a avisos auditivos relativos a estados del paciente que suponen distracciones frecuentes en su desempeño (Castillo et al., 2014; Escribá-Agüir \& Bernabé-Muñoz, 2002; Inoue, Versa, Murassaki, de Melo, \& Matsuda, 2013; Novoa et al., 2005).

En resumen, la Escala Subjetiva de Carga Mental de Trabajo (ESCAM) muestra que es un instrumento que globalmente presenta alta fiabilidad y adecuada validez en una muestra chilena para la evaluación de la carga mental. Entre los aspectos positivos a destacar de este estudio cabe señalar, en primer lugar, que se contó con una muestra amplia y representativa de los distintos grupos profesionales que trabajan en UPC. En segundo lugar, se utilizaron instrumentos que han sido validados previamente en otros estudios internacionales, así como en el contexto cultural y organizacional de Chile. En tercer lugar, el procedimiento de recogida de datos se realizó mediante entrevistas individuales, lo que garantizó una alta tasa de respuesta.

Sin embargo, este estudio presenta también algunas limitaciones. Primero, hubiera sido recomendable utilizar la versión media del SUSESO. ISTAS 21, dado el tamaño de las organizaciones participantes. Segundo, aunque la muestra es representativa, y refleja la distribución habitual de personal a los puestos de trabajo, el tamaño de los grupos profesionales no es equivalente, lo que puede haber influido en los resultados obtenidos.

En futuras investigaciones deberá abordarse la revisión de los ítems o inclusión de otros, como la carga emocional de trabajo, para mejorar la consistencia interna de algunas de las dimensiones de la ESCAM. Asimismo, sería interesante explorar el comportamiento de la escala en profesionales de la salud pertenecientes a distintas unidades hospitalarias. 
Paula Ceballos-Vásquez, Gladys Rolo-González, Estefanía Hernández-Fernaud, Dolores Díaz-Cabrera, Tatiana Paravic-Klijn, Mónica Burgos-Moreno, Omar Barriga

\section{Referencias}

Alvarado, R., Pérez-Franco, J., Saavedra, N., Fuentealba, C., Alarcón, A., Marchetti, N., \& Aranda, W. (2012). Validación de un cuestionario para evaluar riesgos psicosociales en el ambiente laboral en Chile. Revista Médica de Chile, 140(9), 1154-1163. http://dx.doi.org/10.4067/S003498872012000900008

Castillo, I. Y., Torres, N., Ahumada, A., Cárdenas, K., \& Licona, S. (2014). Estrés laboral en enfermería y factores asociados. Cartagena (Colombia). Salud Uninorte, 30(1), 34-43. doi: http://dx.doi. org/10.14482/sun.30.1.4312

Ceballos, P., Paravic, T., Burgos, M., y Barriga, O. (2014). Validación de Escala Subjetiva de Carga Mental de Trabajo en funcionarios/as universitarios. Cienc Enferm, 20(2), 73-82.

Díaz, D., Hernández, E., \& Rolo, G. (2012). Carga mental de trabajo. España: Ed. Síntesis.

Díaz-Cabrera, D., Hernández-Fernaud, E., RoloGonzález, G., Galván, E., Fraile, M., \& Loayssa, G. (2010). Escala Subjetiva de Carga Mental (ESCAM). Tenerife: Instituto Canario de Seguridad Laboral. Recuperado de http://www.gobiernodecanarias.org/cicnt/icasel/documentos/manualcargamental.pdf

Escribá-Agüir, V., \& Bernabé-Muñoz, Y. (2002). Exigencias laborales psicológicas percibidas por médicos especialistas hospitalarios. Gaceta Sanitaria, 16(6), 487- 496. Recuperado de http:// scielo.isciii.es/scielo.php?script $=$ sci_arttext\&pi$\mathrm{d}=$ S0213-91112002000600006\&lng $=e s$

Ferrer, R., \& Dalmau, I. (2014). Ergonomía cognitiva y carga mental. En P. Gil-Monte (Coord.), Manual de Psicosociología aplicada al trabajo y a la prevención de los riesgos laborales (pp. 159-189). Madrid: Pirámide.

Gaillard, A. W. K. (2001). Stress, workload, and fatigue as three biobehavioral states: a general overview. In P.A. Hancock, \& P.A. Desmond (Eds.), Stress, Workload and Fatigue (pp. 623-639). New Jersey: Lawrence Erlbaum Associates.

Gómez-Ortiz, V. (2011). Assessment of psychosocial stressors at work: psychometric properties of the JCQ in Colombian workers. Revista Lati- noamericana de Psicología, 43(2), 329-342. Recuperado de http://www.redalyc.org/articulo. oa?id $=80521287011$

Hacker, W. (2001). Carga mental de trabajo. En Organización Internacional del Trabajo (OIT) (Ed), Enciclopedia de Salud y Seguridad en el Trabajo (Vol. 1, cap. 29, pp. 44-46). Madrid: Instituto Nacional de Seguridad e Higiene en el Trabajo. Ministerio de Trabajo y Asuntos Sociales. (1 1 e edición electrónica en español. Reimpreso de Encyclopaedia of Occupational Health and Safety, fourth edition, por ILO, Ed., 1998, Ginebra). Recuperado de http://www. insht.es/InshtWeb/Contenidos/Documentacion/ TextosOnline/EnciclopediaOIT/tomo1/29.pdf

Inoue, K., Versa, G., Murassaki, A., de Melo, W., \& Matsuda, L. (2013). Estresse ocupacional em enfermeiros intensivistas que prestam cuidados diretos ao paciente crítico. Revista Brasileña de Enfermería, 66(5), 722-729. http://dx.doi.org/10.1590/S0034. 71672013000500013

Meseguer de Pedro, M., Soler, M. I., García-Izquierdo, M., Sáez, M. C., \& Sánchez, J. (2007). Los factores psicosociales de riesgo en el trabajo como predictores del mobbing. Psicothema, 19(2), 225-230. Recuperado de http://www.redalyc.org/articulo. oa? id $=72719207$

Novoa, M. N., Nieto, C., Forero, C., Caycedo, C. E., Palma, M., Montealegre, M. P., Bayona, M., \& Sánchez, C. (2005). Relación entre perfil psicológico, calidad de vida y estrés asistencial en personal de enfermería. Universitas Psychologica, 4(1), 63-75. Recuperado de http://www.redalyc.org/articulo. oa? $\mathrm{id}=64740108$

Patlán, J. (2013). Efecto del burnout y la sobrecarga en la calidad de vida en el trabajo. Estudios Gerenciales, 29(129), 445-455. Recuperado de http://www. redalyc.org/articulo.oa?id=21230026007

Rolo, G., Díaz, D., \& Hernández, E. (2009). Desarrollo de una escala subjetiva de carga mental de trabajo (ESCAM). Rev psicol trab organ, 25(1), 29-37.

Tabachnick, B. G., \& Fidell, L. S. (1989). Using multivariate statistics (2 Ed.). Nueva York: Harper Collins Publishers.

Zeller, R. A., \& Carmines, E. G. (1980). Measurement in the social sciences. Nueva York: Cambridge University Press. 